\title{
Overview of the membrane-associated RING-CH (MARCH) E3 ligase family
}

\author{
Johannes Bauer ${ }^{\mathrm{a}}$, Oddmund Bakke ${ }^{\mathrm{b}}$, J. Preben Morth ${ }^{\mathrm{a}, \mathrm{c}, *}$ \\ a Centre for Molecular Medicine Norway (NCMM), Nordic EMBL Partnership University of Oslo, N-0349 Oslo, Norway \\ ${ }^{\mathrm{b}}$ Department of Biosciences, Centre for Immune Regulation, University of Oslo, N-0316 Oslo, Norway \\ ${ }^{\mathrm{c}}$ Institute for Experimental Medical Research, Oslo University Hospital, N-0424 Oslo, Norway
}

\section{A R T I C L E I N F O}

Article history:

Available online $\mathrm{xxx}$

Keywords:

MARCH E3 ligase

MARCH-1

Ubiquitination

MHC-Il

GxxxG

\begin{abstract}
A B S T R A C T
E3 ligases are critical checkpoints for protein ubiquitination, a signal that often results in protein sorting and degradation but has also been linked to regulation of transcription and DNA repair. In line with their key role in cellular trafficking and cell-cycle control, malfunction of E3 ligases is often linked to human disease. Thus, they have emerged as prime drug targets. However, the molecular basis of action of membrane-bound E3 ligases is still unknown. Here, we review the current knowledge on the membraneembedded MARCH E3 ligases (MARCH-1-6,7,8,11) with a focus on how the transmembrane regions can contribute via GxxxG-motifs to the selection and recognition of other membrane proteins as substrates for ubiquitination. Further understanding of the molecular parameters that govern target protein recognition of MARCH E3 ligases will contribute to development of strategies for therapeutic regulation of MARCH-induced ubiquitination.
\end{abstract}

(C) 2016 Published by Elsevier B.V.

\section{Memoir}

Some of the earliest work on the function of the tRNA initiating protein translation, the N-Formyl-Methionyl-tRNA, was described by Brian FC Clark [1-5], and a follow up study led to the crystal structure of yeast phenylalanine tRNA in the laboratory headed by Aaron Klug [6]. Brian Clark was initially head-hunted to join him at Aarhus University and lead the Department for Biostructural Chemistry, which was the perfect setting for him to continue his vision towards understanding the fundamentals of the genetic code. As a university teacher, Brian was an inspirational speaker and excellent at conveying the message. His teaching always included keeping an open mind and only by combining all the present data, both the structural and the functional, a conclusion could be drawn - an approach that still is integrated into the Biostructural Department today.

\section{The classical ubiquitination pathway}

Ubiquitination is an enzymatic process that involves the addition of an ubiquitin protein to a substrate that usually becomes inactivated followed by degradation in the proteasome; however, several other functions have also been described. For the

\footnotetext{
* Corresponding author at: Centre for Molecular Medicine Norway (NCMM), Nordic EMBL Partnership University of Oslo, N-0349 Oslo, Norway.

E-mail address: j.p.morth@ncmm.uio.no (J. P. Morth).
}

discovery of the ubiquitin-mediated protein degradation pathway, Aaron Ciechanover, Avram Hershko and Irwin Rose were awarded the Nobel prize in 2004 [7]. Over the last two decades, the molecular basis for how the individual components involved with protein ubiquitination interact with each other has been revealed, but it is still far from fully understood.

Protein ubiquitination is initiated by an E1 ubiquitin-activating enzyme (E1) that requires ATP and $\mathrm{Mg}^{2+}$ to catalyse the C-terminal acyl-adenylation of ubiquitin, a reaction that results in an E1 noncovalently bound to adenylated ubiquitin. In the following second reaction step, the catalytic cysteine present in the $\mathrm{E} 1$, attacks the adenylated ubiquitin to form the activated E1-ubiquitin $(\mathrm{E} 1 \sim \mathrm{Ub})$ thioester-bonded complex, with release of AMP [8]. Next, a second ubiquitin molecule is adenylated and bound by the $\mathrm{E} 1 \sim \mathrm{Ub}$ thioester-bonded complex. This is followed by ubiquitin-transfer of its thioester-bonded ubiquitin to an E2 conjugating enzyme (E2), again involving a cysteine residue, to yield a thioester-bonded E2 Ub complex [9]. Finally, an E3 ubiquitin ligase (E3) ensures ubiquitin-coupling from the E2 $\sim$ Ub to a primary amine (i.e. the $\varepsilon$-amine of lysine or the $\mathrm{N}$-terminus) of substrate proteins resulting in a stable isopeptide bond linkage.

E3 ligases are the final catalytic components in the E1-E2-E3 ubiquitination cascade, and are in most cases critical for ubiquitin transfer from the $\mathrm{E} 2 \sim$ Ub complex to its specific substrate proteins. Mono-, multi- and poly-ubiquitination are signals used in protein sorting, signaling, DNA-repair, histone-regulation, and proteasomal/lysosomal degradation (extensively reviewed elsewhere 
Table 1

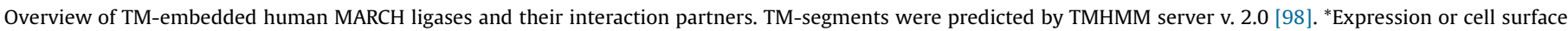
expression of target proteins is downregulated in respective MARCH-expressing cells, but it might not be the direct target.

\begin{tabular}{|c|c|c|c|c|c|}
\hline \multirow[t]{2}{*}{ E3 ligase } & \multicolumn{3}{|c|}{ Interacting protein/Target protein } & \multirow[t]{2}{*}{ Biological significance } & \multirow[t]{2}{*}{ References } \\
\hline & Name & $\begin{array}{l}\text { Number of TM } \\
\text { segments }\end{array}$ & $\begin{array}{l}\text { Experimental evidence for } \\
\text { ubiquitination by given MARCH }\end{array}$ & & \\
\hline \multirow[t]{12}{*}{ MARCH-1 } & Bap31 & 3 & no & controls intracellular transport of MARCH-1 & {$[27]$} \\
\hline & INSR & 2 & yes & regulates insulin signaling & {$[28]$} \\
\hline & CD83 & 1 & no & stabilization of MHC-II in presence of MARCH-1 & [29] \\
\hline & CD86 & 2 & yes & reduces CD86 cell surface expression & [30] \\
\hline & TNR6 (CD95) & - & no* & reduces TNR6 cell surface expression & [31] \\
\hline & CD98 & 12 & no* & $\begin{array}{l}\text { alters the itinerary of clathrin-independent cargo from recycling to } \\
\text { degradation }\end{array}$ & [32] \\
\hline & TfR & 1 & yes & reduces Tf-uptake & [33] \\
\hline & TRAIL-R1 & 1 & yes & regulates cell-surface expression of TRAIL-R1 & [34] \\
\hline & HLA-DM $\alpha$ & 1 & yes & regulates MHC-II antigen presentation and MHC II trafficking & [24] \\
\hline & HLA-DO $\beta$ & 1 & yes & & [35] \\
\hline & HLA-DR $\alpha$ & 1 & yes & & [36] \\
\hline & HLA-DR $\beta$ & 1 & yes & & {$[26,37,38]$} \\
\hline \multirow[t]{7}{*}{ MARCH-2 } & $\beta_{2} \mathrm{AR}$ & 7 & yes & $\begin{array}{l}\text { regulates } \beta_{2} \text { AR cell surface expression and signaling in presence of } \\
\text { carvedilol }\end{array}$ & [39] \\
\hline & STX-6 & 11 & no & inhibits CFTR-mediated autophagy in tumor cells, regulates transferrin & {$[40-42]$} \\
\hline & CAL & - & no & uptake, involved in pathway from endosomes to TGN & \\
\hline & CFTR & 1 & yes & & \\
\hline & DLG1 & - & yes & E3-ligases as regulator of PDZ-proteins or vice versa & [43] \\
\hline & TfR & 1 & no & reduces Tf-uptake & {$[31,42]$} \\
\hline & CD86 & 2 & no* & reduces CD86 cell surface expression & [31] \\
\hline \multirow[t]{4}{*}{ MARCH-3 } & Bap31 & 3 & no & controls intracellular transport of MARCH-3 & [27] \\
\hline & STX-6 & 11 & no & modulates vesicular transport in endosomal recycling, reduces Tf-uptake & {$[44]$} \\
\hline & Fc $\gamma$ RII-b & 1 & yes & downregulates Fc $\gamma$ RII-b after TLR4 activation & [45] \\
\hline & FoxO1 & - & no* & regulates endothelial permeability in response to inflammatory factors & [46] \\
\hline \multirow[t]{7}{*}{ MARCH-4 } & Bap31 & 3 & no & controls intracellular transport of MARCH-4 & {$[27]$} \\
\hline & CD4 & 1 & yes & reduces $\mathrm{CD} 4$ expression & {$[31,47]$} \\
\hline & CD81 & 4 & yes & regulates cell-cell interactions & [27] \\
\hline & STX-4 & 1 & no & modulation of vesicular trafficking & [48] \\
\hline & ALCAM & 1 & no & role in antiviral immunity & \\
\hline & MHC-I & 1 & yes & regulates MHC-I antigen presentation & [31] \\
\hline & Mult1 & 1 & yes & regulates stimulation of immune cells through NKG2D receptor & [49] \\
\hline \multirow{10}{*}{$\begin{array}{l}\text { MARCH-5 } \\
\text { (MITOL) }\end{array}$} & Prkar1a & - & yes & maintains pluripotency of embryonic stem cells in mice & [50] \\
\hline & hFis1 & 1 & yes & regulates mitochondrial morphology & [51-53] \\
\hline & Drp1 & - & yes & & \\
\hline & Mfn1 & - & yes & & \\
\hline & Mfn2 & 1 & no & & \\
\hline & mSOD1 & - & yes & eliminates misfolded and toxic proteins in mitochondria & {$[54-56]$} \\
\hline & $\Delta$ NAT-3Q71 & - & yes & & \\
\hline & $\begin{array}{l}\text { S-nitrosylated } \\
\text { MAP1B-LC1 }\end{array}$ & - & yes & & \\
\hline & TANK & - & yes & enhances signaling of TLR7 in innate immunity & [57] \\
\hline & MAVS & 1 & yes & regulates the immune response against RNA virus infection & {$[58]$} \\
\hline \multirow{21}{*}{$\begin{array}{c}\text { MARCH-6 } \\
\text { (TEB4) } \\
\text { MARCH-8 } \\
\text { (c-MIR) }\end{array}$} & SM & 4 & yes & regulates sterol homeostasis & [59] \\
\hline & $\mathrm{DIO} 2$ & - & yes & regulates thyroid hormone levels & {$[60]$} \\
\hline & Bap31 & 3 & $?$ & $\begin{array}{l}\text { targets MARCH- } 8 \text { to the cell surface, controls intracellular transport of } \\
\text { MARCH-8 }\end{array}$ & [27] \\
\hline & CD44 & 1 & yes & regulates cell-cell interactions & \\
\hline & CD81 & 4 & yes & & \\
\hline & CD86 & 2 & yes & regulates immune response & [61] \\
\hline & TNR6 (CD95) & - & no* & reduces TNR6 cell surface expression & [31] \\
\hline & Il1RAP & 1 & yes & regulates IL-1 $\beta$-induced signaling pathways & {$[62,63]$} \\
\hline & STX-4 & 1 & no & modulation of vesicular trafficking & {$[48]$} \\
\hline & TfR & 1 & yes & regulates Tf-uptake & [33] \\
\hline & E-cadherin & 1 & yes & modulates cell adhesion in zebrafish embryos & [64] \\
\hline & $\begin{array}{l}\text { viral envelope } \\
\text { glycoproteins }\end{array}$ & ? & no* & reduces virion incorporation of envelope glycoproteins & [65] \\
\hline & HLA-DO $\beta$ & 1 & yes & regulates MHC-II antigen presentation & [35] \\
\hline & HLA-DR $\alpha$ & 1 & yes & & [66] \\
\hline & MHC-II & 1 & yes & & {$[67]$} \\
\hline & $\beta$-chain & & & & \\
\hline & MHC-I & 1 & no* & alters the itinerary of clathrin-independent cargo from recycling to & [32] \\
\hline & CD98 & 12 & yes & degradation & \\
\hline & CD44 & 1 & no* & & \\
\hline & TRAIL-R1 & 1 & yes & regulates cell-surface expression of TRAIL-R1 & [34] \\
\hline & CD83 & 1 & no & stabilization of MHC-II in presence of MARCH-8 & [25] \\
\hline
\end{tabular}


Table 1 (Continued)

\begin{tabular}{|c|c|c|c|c|c|}
\hline \multirow[t]{2}{*}{ E3 ligase } & \multicolumn{3}{|c|}{ Interacting protein/Target protein } & \multirow[t]{2}{*}{ Biological significance } & \multirow[t]{2}{*}{ References } \\
\hline & Name & $\begin{array}{l}\text { Number of TM } \\
\text { segments }\end{array}$ & $\begin{array}{l}\text { Experimental evidence for } \\
\text { ubiquitination by given MARCH }\end{array}$ & & \\
\hline \multirow[t]{18}{*}{ MARCH-9 } & Bap31 & 3 & no & controls intracellular transport of MARCH-9 & {$[27]$} \\
\hline & ALCAM & 1 & no & role in antiviral immunity & {$[48]$} \\
\hline & MHC-I & 1 & yes & regulates MHC-I antigen presentation & [31] \\
\hline & HLA-DM $\alpha$ & 1 & yes & regulates MHC-II antigen presentation & [24] \\
\hline & HLA-DO $\beta$ & 1 & yes & & [35] \\
\hline & HLA-DQA & 1 & no* & & [68] \\
\hline & HLA-DQB & 1 & no* & & \\
\hline & Mult1 & 1 & yes & regulates stimulation of immune cells through NKG2D receptor & [49] \\
\hline & ICAM1 & 1 & yes & regulates lymphocyte function & [69] \\
\hline & Fc $\gamma$ RII-b & 1 & no* & regulates B-cell receptor signaling & [68] \\
\hline & PTPRA & 2 & no* & modulates the immune response & \\
\hline & PTPRJ & 1 & no* & & \\
\hline & SLAM & 2 & yes & & \\
\hline & ILT-2 & 1 & no* & & \\
\hline & VAMP8 & 1 & no* & regulates endosomal vesical fusion & \\
\hline & TMEM2 & 1 & no* & involved in cell adhesion & \\
\hline & Plexin-C1 & 2 & no* & involved in integrin-mediated adhesion of dendritic cells & \\
\hline & FCRL2 & 1 & no* & role in B-cell development & \\
\hline \multirow{4}{*}{$\begin{array}{l}\text { MARCH- } \\
\quad 11\end{array}$} & CD4 & 1 & yes & regulates CD4 levels & [47] \\
\hline & Veli & - & no & sorts proteins in pathway between TGN and multivesicular bodies & {$[31,47]$} \\
\hline & $\mu 1$-adaptin & - & no & & \\
\hline & SAMT1 & 4 & yes & involved in mice spermatogenesis & [70] \\
\hline
\end{tabular}

Abbreviations:

B2AR: beta-2 adrenergic receptor;

ALCAM: activated leukocyte cell adhesion molecule (CD166);

Bap31: B-cell receptor-associated protein 31;

CAL: CFTR-associated ligand (alternative name: GOPC: Golgi-associated PDZ and coiled-coil motif-containing protein);

CD: cluster of differentiation;

CFTR: cystic fibrosis transmembrane conductance regulator;

DIO2: type II iodothyronine deiodinase;

DLG1:disks large homolog 1;

$\Delta$ NAT-3Q71: N-terminal truncated pathogenic ataxin-3 with a 71-glutamine repeat;

Drp1: dynamin-related protein 1;

FCRL2: Fc receptor-like protein 2;

Fc $\gamma$ RII-b: IgG Fc receptor II-b;

FoxO1: forkhead box protein 1;

hFis1: human mitochondrial fission 1 protein;

ICAM1: intracellular adhesion molecule 1;

IL1RAP: interleukin 1 receptor accessory protein;

ILT-2: immunoglobulin-like transcript 2;

MAVS: mitochondrial antiviral-signaling protein;

Mfn: mitofusin;

MHC: major histocompatibility complex;

mSOD1: mutant superoxide dismutase 1;

Mult1: murine ULBP-like transcript 1;

NKG2D: natural killer cell group 2D;

PDZ: PSD95/DLG/ZO-1 (post-synaptic density protein 95 / Drosophila lethal disk large tumor suppressor protein / zona occluden-1);

Prka1a : cAMP-dependent protein kinase type I-alpha regulatory subunit;

PTPRA: receptor-type tyrosine-protein phosphatase alpha;

PTPRJ: receptor-type tyrosine-protein phosphatase eta;

SAMT: spermatogenesis-associated multicopy transmembrane protein 1;

SLAM: signaling lymphocytic activation molecule;

SM: squalene monooxygenase;

MAP1B-LC1: microtubule-associated protein 1B light chain 1;

STX: syntaxin;

TANK: TRAF family member associated NF- $\kappa \mathrm{B}$ activator;

Tf: transferrin;

TfR: transferrin receptor protein 1 ;

TGN: trans-Golgi network;

TLR: Toll-like receptor;

TM: transmembrane;

TMEM2: transmembrane protein 2;

TNR6: tumor necrosis factor receptor superfamily member 6 (=FAS1);

TRAIL-R1: tumor necrosis factor-related apoptosis-inducing ligand receptor 1;

VAMP: vesicle-associated membrane protein. 
$[10,11])$. Most E3 ligases are grouped into the HECT-E3 (homologous to E6-AP carboxy terminus) or the larger RING-E $\overline{3}$ (really interesting new gene) ligase family [11]. RING-E3 ligases directly mediate ubiquitin transfer from an E2 to a specific substrate, while the HECT-E3s transfer ubiquitin via an E3 Ub thioester intermediate [12]. The main functions of RING-E3s are to provide the scaffold that allows simultaneous binding of E2 and the substrate protein, and in addition to induce a "closed" E2 $\sim$ Ub conformation that is primed for attack by the substrate nucleophile [13]. More than 600 RING-E3s have been found in humans and they outnumber the E1 and E2 proteins by far, with only two E1s and nearly forty E2s in humans [13]. Thus, E3 ligases impose, to a large extent, specificity onto the ubiquitination pathway and are therefore considered prime drug targets [14]. Consistent with their fundamental role in orchestrating cellular trafficking of target proteins, malfunction and faulty regulation of E3s is often associated with human disease including viral infections [15], neurodegenerative disorders [16,17] and cancer [14].

\section{MARCH E3 ligases}

A specific family of eukaryotic E3 ligases that has received additional attention recently consists of the membrane-associated RING-CH (MARCH) proteins. Initially, two viral MAR $\bar{C}$ H-homologue gene products were discovered in Kaposi's sarcoma (KS)associated herpesvirus (KSHV), and subsequently named K3 (kK3) and K5 (kK5). Later, kK3- and kK5-related E3s were found in poxviruses and a K3-related E3 ligase was discovered in myxomavirus (termed mK3) [18]. These viral MARCH E3s help escape from host defence mechanisms by down-regulating major histocompatibility complex (MHC) class I (MHC-I) antigen presentation and are now thought to originate from the human MARCH proteins due to their overlapping substrate spectrum and structural similarity [18-20]. Interestingly, viral MARCHs are often able to ubiquinate their targets on non-lysine residues, such as cysteine, serine and threonine residues [19,21,22].

The human MARCH family comprises eleven members (termed MARCH-1 to 11), of which nine are transmembrane proteins. In this review, we focus on the role of the membrane-spanning MARCH proteins and how their transmembrane regions can mediate interactions with their target proteins. Therefore, the cytosolic MARCH-7 and MARCH-10 proteins are not regarded here (but have been reviewed elsewhere $[19,23])$. MARCH proteins show overlapping substrate specificity (Table 1), and this can be rationalised by phylogenetic analysis (Fig. 1). Even though some MARCHs ubiquitinate the same substrate, they might do so in different cellular compartments, at different time points, and with a different ubiquitination pattern resulting in differential sorting of their target proteins into different cellular pathways[24-26].

\section{Architecture of human MARCH proteins}

Human MARCH proteins share the RINGv domain ( $6 \mathrm{kDa})$ that coordinates two $\mathrm{Zn}^{2+}$ ions in a cross-braced manner for recognition of a cognate E2 protein. MARCH-7 and MARCH-10 are soluble nonmembrane associated E3 ligases with the RINGv domain at the C-terminus, whereas the other family members are membraneembedded proteins with two transmembrane (TM) helices predicted in MARCH-1/8, MARCH-2/3, and MARCH-4/9/11, four in MARCH-5 and 14 in MARCH-6 (Fig. 1).

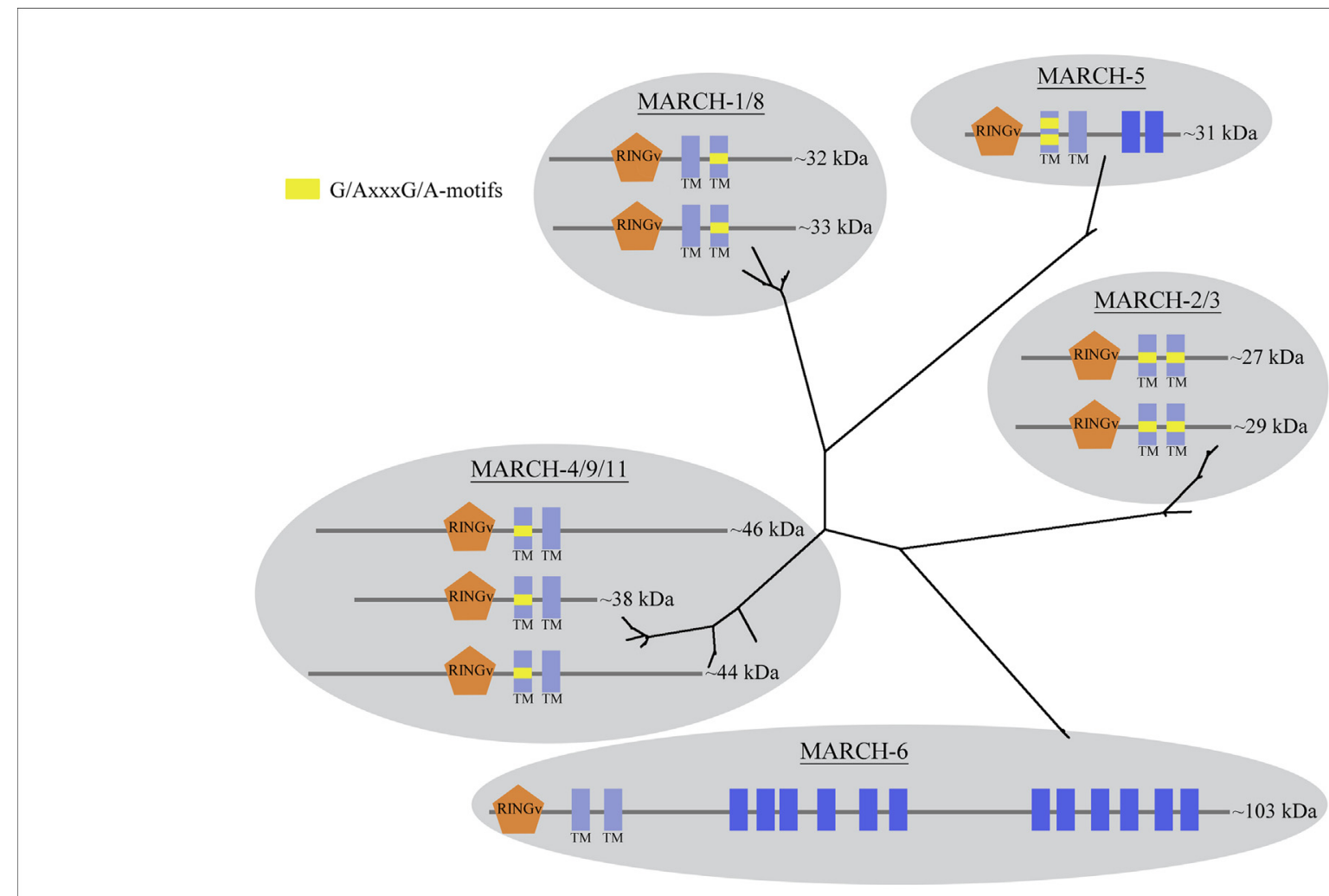

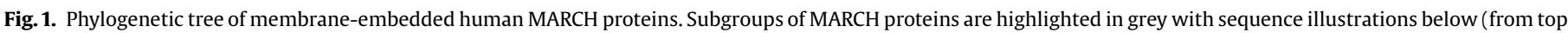

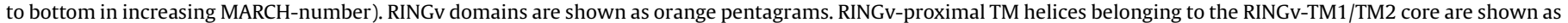

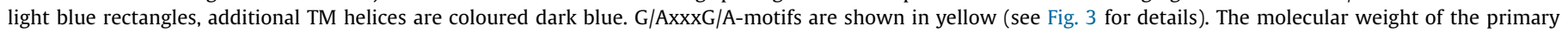
structure is given on the right of the respective MARCH protein. Phylogenetic analysis was calculated with MEGA7 [99] using the MARCH-sequences given in Fig. 3. 
The RINGv domain is always placed N-terminal to the first TM helix at a distance of approximately 13-36 residues from the first TM helix. TM1 and TM2 are themselves connected via a 13-27 amino acid (aa) long luminal loop (L1). This RINGv-TM1/2 segment is often confined either by disordered stretches (i) before the RINGv domain (MARCH-1/8), (ii) after the TM2 helix (MARCH-6), (iii) on both sides (MARCH-4/9/11), or (iv) by sequence boundaries, i.e. that the RINGv is already placed at the very $\mathrm{N}$-terminus of the sequence (MARCH-5 and MARCH-6). Thus, the common structural element in membrane-embedded MARCH proteins is a cytosolic RINGv domain followed by two transmembrane helices (Fig. 1).

Consistently, the sequence conservation of the subgroup members is most pronounced within this RINGv-TM1/2 segment when compared to the entire sequence. (MARCH-1/8: $89 \%$ vs. $65 \%$, MARCH-2/3: 76\% vs. 65\%, MARCH-4/9: 91\% vs. 67\%, MARCH-4/11: $75 \%$ vs. $49 \%$, MARCH-9/11: $72 \%$ vs. $49 \%$ ). Although sorting motifs are not necessarily included in this RINGv-TM1/TM2 segment, it is likely to be sufficient to ensure E3 ligase activity. This includes E3-binding to both a cognate E2-conjugating enzyme and a substrate protein followed by substrate ubiquitination.

\section{Oligomerisation of MARCH proteins}

RING-E3 ligases are often found both as monomers and homodimers, but heterodimers have also been reported [11]. However, the dimerisation interfaces can vary, and are grouped in two distinct types, the RINGv-mediated interfaces and the nonRINGv mediated interfaces [13]. E3 ligases have also been shown to exist as higher oligomers, as revealed by negative-stain electron microscopy for the tetrameric pre-mRNA splicing factor Prp19p [71]. Prp19p is a soluble U-box E3 ligase that is structurally related to RING-E3 ligases but lacks the eight canonical $\mathrm{Zn}^{2+}$-coordinating residues [72].

Oligomerisation for MARCH proteins was first shown for the viral MARCH kK5 by co-immunoprecipitation and the responsible region could be traced to the TM1-L1-TM2 fragment [73]. Next, MARCH-9 was shown to form oligomers with a truncated MARCH9 protein lacking the RINGv domain by co-immunoprecipitation [69], also indicating that the RINGv-domain is not necessary for oligomerisation in MARCH proteins. Co-immunoprecipitation showed homo-oligomerisation for MARCH-1 and MARCH-8, as well as possible hetero-oligomerisation for MARCH-1/MARCH-8 and MARCH-1/MARCH-9 [74]. Furthermore, MARCH-1 homo- oligomerisation and MARCH-1/MARCH-8 hetero-oligomerisation was confirmed by bioluminescence resonance energy transfer (BRET) [74]. Importantly, these experiments do not exclude the formation of higher oligomers than dimers. Interestingly, co-immunoprecipitation studies with MARCH-1 chimeras, which either had TM1, TM2 or both TM helices replaced by MARCH-9 TM helices, revealed that oligomerisation with MARCH-1 was only rescued when both TM1 and TM2 were exchanged [75]. In conclusion, the oligomerisation of MARCH proteins is most likely only mediated by the TM helices with little or no contribution from the RINGv-domains.

\section{Ubiquitination of MARCH target proteins}

The RING-E3 ligase activity requires MARCH proteins to simultaneously bind a cognate E2 conjugating enzyme and a substrate protein. Ubiquitination of MARCH target proteins takes place on the cytosolic side $[22,36,76]$ inline with binding of E2 proteins to the cytosolic RINGv domain.

Both, the kK3 and kK5-mediated ubiquitination leads to rapid internalisation of target proteins followed by lysosomal degradation, similar to that seen for MARCH-1/8 [77]. Ubiquitination activity of kK3 and kK5 depends on the positioning of the targeted lysine (or cysteine) residues [22]. The positioning of the lysine residue on the substrate is in turn determined by the interaction site of the substrate with the respective E3 ligase.

Although mK3 targets its substrates for proteasomal degradation, it is noteworthy that mK3-mediated ubiquitination seems to be independent of any secondary structure or the amino acid sequence in the cytosolic tail of an MHC-I heavy chain, as long as two lysine, serine or threonine residues are present at the C-terminus of an engineered glycine-rich tail [20]. This suggests that the cytosolic tail of the substrate is not involved in mK3binding and in turn indicates that the interface in $\mathrm{mK} 3$ is located within the TM helices or the luminal L1-loop. However, it was also suggested that $\mathrm{mK} 3$ binds MHC-I heavy chains indirectly via heterodimer TAP-1 and TAP-2, peptide transporters that are part of the MHC-I peptide loading complex [78]. Experimentally validated interaction partners of membrane-embedded MARCHs are mostly membrane proteins themselves (Table 1). Interestingly, these MARCH-E3 substrates vary in their number of TM segments and the sizes of their cytosolic and luminal regions. It is therefore tempting to speculate that the MARCH TM helices fulfil two

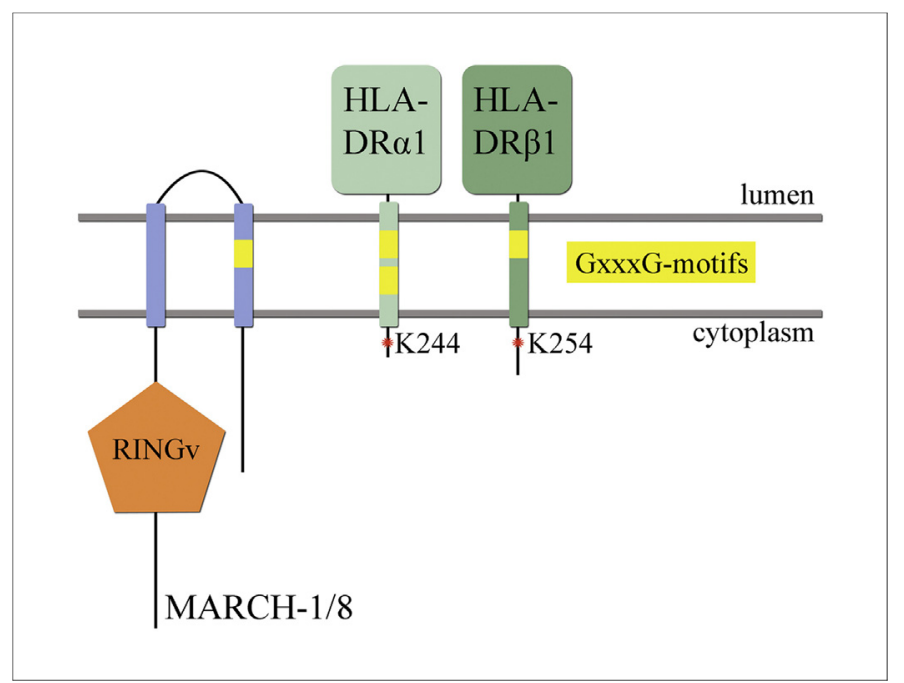

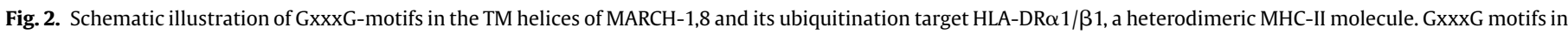

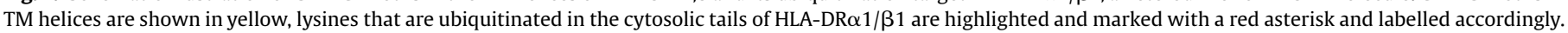


functions: to provide interfaces for both oligomerisation and substrate recognition.

Thoroughly characterised substrates for human MARCH-1/8 are the MHC-II molecules, such as HLA-DR $\alpha 1 / \beta 1$, which become ubiquitinated on their $\sim 15$ amino acid long cytosolic tails of the $\alpha$ - and $\beta$-chains $[36,76]$. HLA-DR $\alpha / \beta$ are heterodimeric, mainly luminal proteins with one TM-helix each and a short cytosolic tail. In contrast, the E2-binding RINGv domains of MARCH-1/8 are located in the cytoplasm (Fig. 2). Thus, interaction between HLA-DR $\alpha / \beta$ and MARCH-1/8 can in principle be mediated by the TM regions, the luminal loop, and/or the cytosolic linker between RINGv and TM1 of MARCH-1/8.

Formation of MHC-II heterodimers is mediated by GxxxG motifs in their transmembrane helices $[79,80]$. The functional importance of the GxxxG mediated HLA-DR $\alpha / \beta$ interface was recently highlighted, when it was pointed out that the two GxxxG
TM1

MARCH-1/8
sp|Q8TCQ1|MARCH1_Human
sp|Q6NZQ8|MARCH1_Mouse
tr|F1QSH0|MARCH1_Zebrafish
tr|F7D0P9|MARCH1_Xenopus
tr|F1MM17|MARCH1_Bos taurus
sp|Q5T0T0|MARCH8_Human
sp|Q9DBD2|MARCH8_Mouse
tr|A0A0R4IAR7|MARCH8_Zebrafish
sp|Q28IK8|MARCH8_Xenopus
sp|Q0VD59|MARCH8 Bos taurus

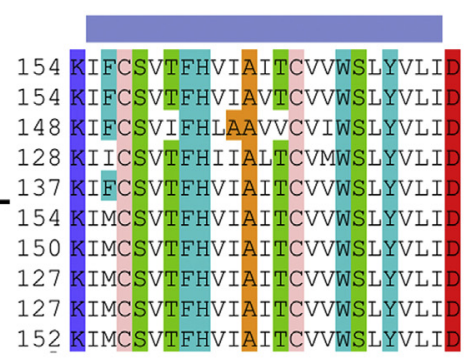

MARCH-2/3

sp |Q9P0N8|MARCH2_Human sp |Q99M02 | MARCH2 Mouse sp |Q1LVZ2 | MARCH2_Zebrafish sp | Q1LVZ2 | MARCH2_Xenopus sp|Q32L65 |MARCH2 Bos taurus sp|Q86UD3 |MARCH3_Human sp | Q8BRX9 | MARCH3 Mouse sp |Q68FA7 | MARCH3 Xenopus sp|A0JN69|MARCH3 Bos taurus

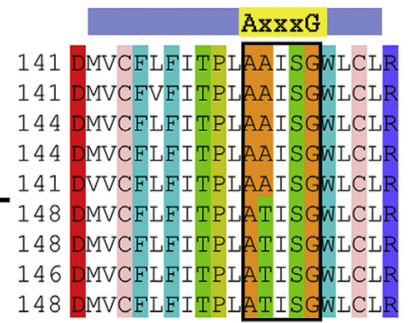

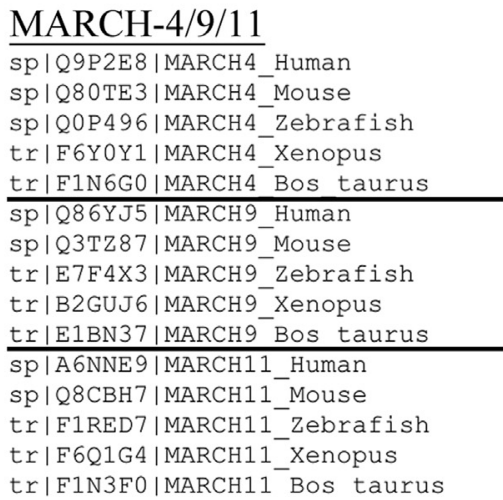

\section{MARCH-5}

spIQ9NX47|MARCH5 Human sp IQ3KNM2 | MARCH5 Mouse sp|Q6NYK8|MARCH5_Zebrafish sp|Q6GM4 4 |MARCH5_Xenopus spIQ3ZC24|MARCH5 Bos taurus
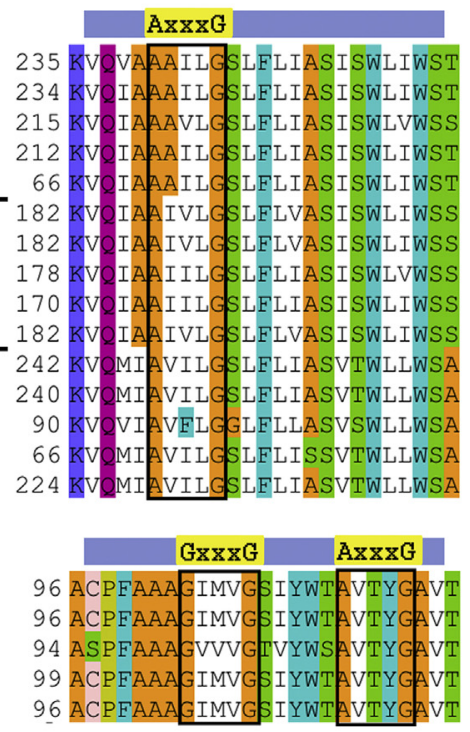

\section{MARCH-6}

sp|060337|MARCH6 Human sp|Q6ZQ89|MARCH6_Mouse tr|E9QDQ9|MARCH 6 Zebrafish $\operatorname{tr|A2RV74|MARCH6-Xenopus~}$ tr|F1MHF0|MARCH 6 Bos taurus

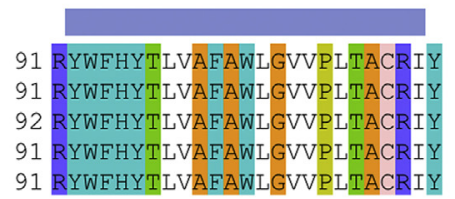

\section{TM2}

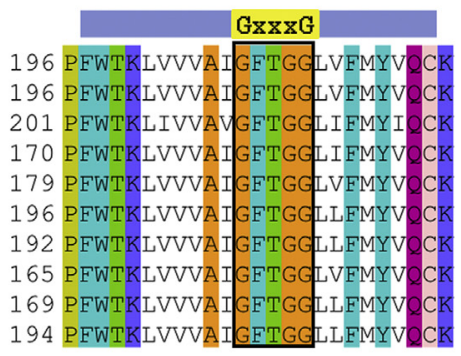

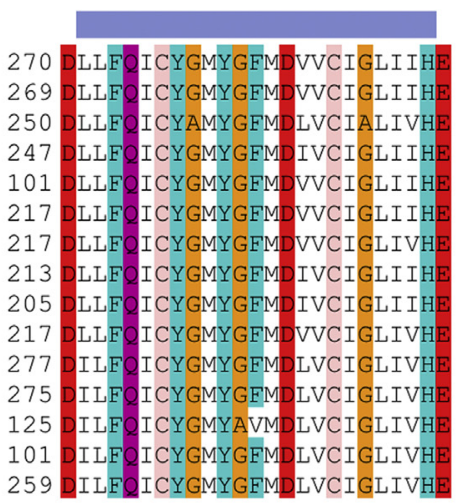
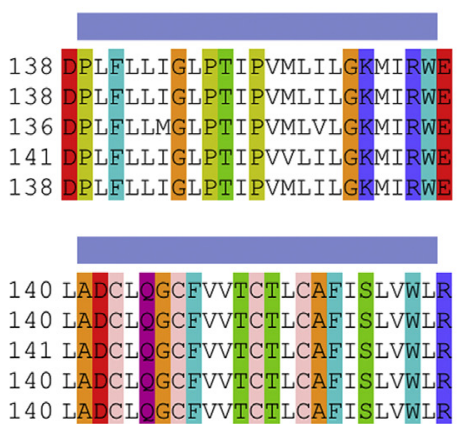

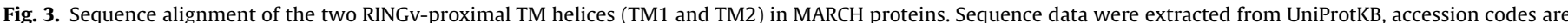

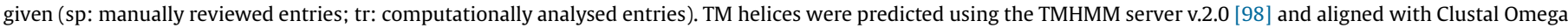

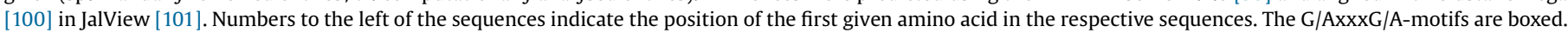

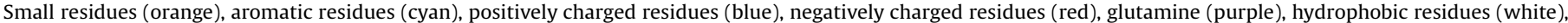

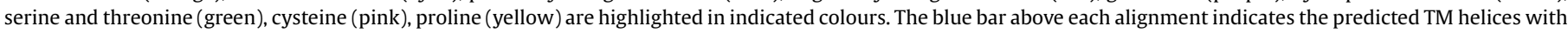
the respective G/AxxxG/A motif highlighted. A black line separates the aligned MARCH families. 
motifs in the TM region of HLA-DR $\alpha$ allow for two positions that HLA-DR $\beta$ can recognise with its single GxxxG motif, also located in the TM region. Importantly, the two different vertical positions of TM helices forming the HLA-DR $\alpha / \beta$ interface likely have longrange implications for binding of antigens and their presentation to T-cells [81,82]. Interestingly, GxxxG motifs are also found in MARCH TM helices and might therefore also serve as recognition points for the MHC-molecules and other substrate proteins (Fig. 2).

The GxxxG-motif

In soluble proteins, the GxxxG-motif is mainly found in helix-helix interactions and, in a few cases, also in helix$\beta$-strand interactions [83]. Furthermore, in amyloid fibrils GxxxG motifs occur in parallel-stranded $\beta$-sheets forming grooves on the $\beta$-sheet surface [84]. Interestingly, GxxxGmotifs are most often found in TM helices of integral membrane proteins where they can mediate oligomerisation, intramolecular helix-helix interactions, and/or protein-protein interactions [85-87]. Although GxxxG is one of the most abundant identified motifs in TM helix-helix interactions, its presence per se is only a weak predictor for a physical interaction $[88,89]$. The key to a specific interaction likely includes the sequence context of the GxxxG-motif [90,91]. However, it is unclear whether the surrounding amino acid composition in such a structural motif can be used to predict involvement in dimerisation or proteinprotein interactions [88]. However, a central placement of the GxxxG motif within a TM helix does result in a stronger dimerisation interface [92].

Generally, glycines (and other small residues such as alanine) present in a helical GxxxG-motif that are facing the same side of the helix form a uniform surface that enables direct contact with a second helix. Crystal structures of multipass membrane proteins have demonstrated that GxxxG-motifs are used in various constellations to pack TM helices. In the T. thermophilus transhydrogenase domain II [93] or the sodium-proton antiporter NhaA [94] some GxxxG-motifs (and extended repeats thereof) are facing towards each other (in some cases with a shifted register), whereas other GxxxG-motifs are only present in one of the interacting helices, but still forming a dimer interface with the neighbouring TM helix. In the sodium-potassium pump [95] a variant of the GxxxG-motif (AxxxA) is found at the TM interface between the $\alpha$ and $\gamma$-subunits. This AxxxA-motif is present in both interacting helices and both motifs are facing towards each other (again a shift in register is observed). In the $\beta-/ \alpha$-subunit binding interface, the extended motif GxxxGxxxG in the $\beta$-subunit faces the lipid environment and thus away from the interface [95]. This might allow for binding of interaction partners or simply demonstrates that not all GxxxG-motifs are involved in direct helix-helix interactions.

\section{The GxxxG-motif in MARCH-1/8}

Sequence alignment of MARCH-1/8 homologues reveals a strongly conserved GxxxG-motif in the central part of the TM2 helix (Fig. 3). A third glycine residue is placed at the $n+3$ position, which might allow for the accommodation of bulkier sidechains or intertwined helices. The canonical GxxxG-motif is placed centrally within the TM2 helix and it would not come as a surprise if this motif is involved in helix-helix interactions either by mediating oligomerisation or substrate recognition. However, structural information is needed to confirm this hypothesis. Interestingly, TM1 does not contain any GxxxG or related motif.

In addition to the motif found in TM2, a second GxxxG-motif is present in the L1-loop in MARCH-1/8. The motif is less conserved within MARCH-1/8 homologues and is present in the middle of the L1-loop. The L1-loop is generally rich in amino acid residues with high turn propensities [96], suggesting lack of secondary structure in this loop. However, as the length of the L1-loop in e.g. human MARCH-1 with 14 amino acid residues is longer than needed for just a simple turn, one could speculate that the motif-surrounding residues create flexibility and that the motif engages interaction partners on the luminal side, e.g. via parallel extension of a $\beta$-sheet.

\section{The GxxxG-motif in the other MARCH proteins}

The GxxxG-motif is also present in TM helices of the other MARCH proteins (Fig. 3). In MARCH-2/3, the GxxxG-variants are found both in TM1 and TM2. Interestingly, the two motifs (AxxxG in TM1 and AxxxA in TM2) are positioned at similar distance from the luminal membrane. This would allow for a classical dimerisation interface involving the GxxxG-like motifs in MARCH-2/3.

MARCH-4/9/11 have an AxxxG-motif in TM1 close to the cytoplasmic membrane, while there is no such motif in TM2. MARCH-5 even has two GxxxG-motif variants (GxxxG and AxxxG) in TM1, but none in TM2. Interestingly, mutation of this GxxxG motif to LxxxL prevented oligomerisation of MARCH-5 molecules, while the same mutation of another GxxxG motif in the L1-loop between TM1 and TM2 did not interfere with oligomerisation [97]. March-6 does not contain any GxxxG- or related motifs in TM1, but a CxxxS motif is found in TM2. Cysteines and serines are in this context also defined as small residues that can promote helix-helix interactions [88].

\section{Conclusions}

A large number of membrane proteins use GxxxG-motifs (and variants thereof) in their TM helices for intra- and intermolecular recognition of other TM helices. Here we describe that this GxxxGmotif is also found in different variations in all membraneembedded MARCH E3 ligases. Presence, number and positioning of these motifs in the two core TM-helices (TM1 and TM2) differ between the phylogenetic MARCH subgroups. However, when the motif is present it is always strongly conserved, suggesting a role in oligomerisation and/or substrate recognition for these MARCHproteins. However, structural data on the MARCH proteins in context with the TM helices is completely lacking, but would be beneficial for the design of drugs that regulate $\mathrm{MARCH}$-induced ubiquitination.

\section{Acknowledgements}

The research leading to these results has received funding from the European Union Seventh Framework Programme (FP7PEOPLE-2013-COFUND) under grant agreement no 609020 Scientia Fellows (JB) and the Research Council of Norway (NFRgrant 221444) (JB, JPM, OB).

\section{References}

[1] Clark BF, Marcker KA. N-formyl-methionyl-sigma-ribonucleic acid and chain initiation in protein biosynthesis. polypeptide synthesis directed by a bacteriophage ribonucleic acid in a cell-free system. Nature 1966;211:37880

[2] Clark BF, Marcker KA. The role of N-formyl-methionyl-sRNA in protein biosynthesis. J Mol Biol 1966;17:394-406.

[3] Clark BF, Marcker KA. How proteins start. Sci Am 1968;218:36-42.

[4] Cory S, Marcker KA, Dube SK, Clark BF. Primary structure of a methionine transfer RNA from escherichia coli. Nature 1968;220:1039-40.

[5] Dube SK, Marcker KA, Clark BF, Cory S. Nucleotide sequence of N-formylmethionyl-transfer RNA. Nature 1968;218:232-3.

[6] Robertus JD, Ladner JE, Finch JT, Rhodes D, Brown RS, Clark BF, et al. Structure of yeast phenylalanine tRNA at 3 A resolution. Nature 1974;250:546-51.

[7] [Anonymous]. The nobel prize in chemistry 2004. nobelprize.org. nobel media AB 2014. web. 29 jul 2016. < http://www.nobelprize.org/nobel_prizes/ chemistry/laureates/2004/>. 
[8] Schulman BA, Harper JW. Ubiquitin-like protein activation by E1 enzymes: the apex for downstream signalling pathways. Nat Rev Mol Cell Biol 2009;10:319-31.

[9] Olsen SK, Lima CD. Structure of a ubiquitin E1-E2 complex: insights to E1-E2 thioester transfer. Mol Cell 2013;49:884-96.

[10] Hoeller D, Hecker CM, Dikic I. Ubiquitin and ubiquitin-like proteins in cancer pathogenesis. Nat Rev Cancer 2006;6:776-88.

[11] Deshaies RJ, Joazeiro CA. RING domain E3 ubiquitin ligases. Annu Rev Biochem 2009;78:399-434.

[12] Petroski MD, Deshaies RJ. Function and regulation of cullin-RING ubiquitin ligases. Nat Rev Mol Cell Biol 2005;6:9-20.

[13] Metzger MB, Pruneda JN, Klevit RE, Weissman AM. RING-type E3 ligases: master manipulators of E2 ubiquitin-conjugating enzymes and ubiquitination. Biochim Biophys Acta 2014;1843:47-60.

[14] Morrow JK, Lin H, Sun S, Zhang S. Targeting ubiquitination for cancer therapies. Future Med Chem 2015;7:2333-50.

[15] Tada T, Zhang Y, Koyama T, Tobiume M, Tsunetsugu-Yokota Y, et al. MARCH8 inhibits HIV-1 infection by reducing virion incorporation of envelope glycoproteins. Nat Med 2015;21:1502-7.

[16] Ardley HC, Robinson PA. The role of ubiquitin-protein ligases in neurodegenerative disease. Neurodegenerative Dis 2004;1:71-87.

[17] Shimura H, Hattori N, Kubo S, Mizuno Y, Asakawa S, et al. Familial parkinson disease gene product, parkin, is a ubiquitin-protein ligase. Nat Genet 2000;25:302-5.

[18] Fruh K, Bartee E, Gouveia K, Mansouri M. Immune evasion by a novel family of viral PHD/LAP-finger proteins of gamma-2 herpesviruses and poxviruses. Virus Res 2002;88:55-69.

[19] Wang X, Herr RA, Hansen T. Viral and cellular MARCH ubiquitin ligases and cancer. Semin Cancer Biol 2008;18:441-50.

[20] Herr RA, Harris J, Fang S, Wang X, Hansen TH. Role of the RING-CH domain of viral ligase $\mathrm{mK} 3$ in ubiquitination of non-lysine and lysine MHC I residues. Traffic 2009;10:1301-17.

[21] Cadwell K, Coscoy L. Ubiquitination on nonlysine residues by a viral E3 ubiquitin ligase. Science 2005;309:127-30.

[22] Cadwell K, Coscoy L. The specificities of kaposi's sarcoma-associated herpesvirus-encoded E3 ubiquitin ligases are determined by the positions of lysine or cysteine residues within the intracytoplasmic domains of their targets. J Virol 2008;82:4184-9.

[23] Samji T, Hong S, Means RE. The membrane associated RING-CH proteins: a family of E3 ligases with diverse roles through the cell. Int Sch Res Not 20142014: Article ID 637295

[24] Jahnke M, Trowsdale J, Kelly AP. Ubiquitination of human leukocyte antigen (HLA)-DM by different membrane-associated RING-CH (MARCH) protein family E3 ligases targets different endocytic pathways. J Biol Chem 2012;287:7256-64.

[25] von Rohrscheidt J, Petrozziello E, Nedjic J, Federle C, Krzyzak L, et al. Thymic CD4 T cell selection requires attenuation of March8-mediated MHCII turnover in cortical epithelial cells through CD83. J Exp Med 2016;213:168594.

[26] Walseng E, Furuta K, Bosch B, Weih KA, Matsuki Y, et al. Ubiquitination regulates $\mathrm{MHC}$ class II-peptide complex retention and degradation in dendritic cells. Proc Natl Acad. Sci U S A 2010;107:20465-70.

[27] Bartee E, Eyster CA, Viswanathan K, Mansouri M, Donaldson JG, Fruh K. Membrane-associated RING-CH proteins associate with Bap31 and target CD81 and CD44 to lysosomes. PLoS One 2010;5:e15132.

[28] Nagarajan A, Petersen MC, Nasiri AR, Butrico G, Fung A, et al. MARCH1 regulates insulin sensitivity by controlling cell surface insulin receptor levels. Nat Commun 2016;7:12639.

[29] Tze LE, Horikawa K, Domaschenz H, Howard DR, Roots CM, et al. CD83 increases MHC II and CD86 on dendritic cells by opposing IL-10-driven MARCH1-mediated ubiquitination and degradation. J Exp Med 2011;208:149-65.

[30] Corcoran K, Jabbour M, Bhagwandin C, Deymier MJ, Theisen DL, Lybarger L. Ubiquitin-mediated regulation of $\mathrm{CD} 86$ protein expression by the ubiquitin ligase membrane-associated RING-CH-1 (MARCH1). J Biol Chem 2011;286:37168-80.

[31] Bartee E, Mansouri M, Hovey Nerenberg BT, Gouveia K, Fruh K. Downregulation of major histocompatibility complex class I by human ubiquitin ligases related to viral immune evasion proteins. J Virol 2004;78:1109-20.

[32] Eyster CA, Cole NB, Petersen S, Viswanathan K, Fruh K, Donaldson JG. MARCH ubiquitin ligases alter the itinerary of clathrin-independent cargo from recycling to degradation. Mol Biol Cell 2011;22:3218-30.

[33] Fujita H, Iwabu Y, Tokunaga K, Tanaka Y. Membrane-associated RING-CH (MARCH) 8 mediates the ubiquitination and lysosomal degradation of the transferrin receptor. J Cell Sci 2013;126:2798-809.

[34] van de Kooij B, Verbrugge I, de Vries E, Gijsen M, Montserrat V, et al. Ubiquitination by the membrane-associated RING-CH-8 (MARCH-8) ligase controls steady-state cell surface expression of tumor necrosis factor-related apoptosis inducing ligand (TRAIL) receptor 1. J Biol Chem 2013;288:6617-28.

[35] Jahnke M, Trowsdale J, Kelly AP. Ubiquitination of HLA-DO by MARCH family E3 ligases. Eur J Immunol 2013;43:1153-61.

[36] Lapaque N, Jahnke M, Trowsdale J, Kelly AP. The HLA-DRalpha chain is modified by polyubiquitination. J Biol Chem 2009;284:7007-16.

[37] Matsuki Y, Ohmura-Hoshino M, Goto E, Aoki M, Mito-Yoshida M, et al. Novel regulation of MHC class II function in B cells. EMBO J 2007;26:846-54.
[38] De Gassart A, Camosseto V, Thibodeau J, Ceppi M, Catalan N, et al. MHC class II stabilization at the surface of human dendritic cells is the result of maturation-dependent MARCH I down-regulation. Proc Natl Acad Sci U S A 2008;105:3491-6.

[39] Han SO, Xiao K, Kim J, Wu JH, Wisler JW, et al. MARCH2 promotes endocytosis and lysosomal sorting of carvedilol-bound beta(2)-adrenergic receptors. J Cell Biol 2012;199:817-30.

[40] Cheng J, Guggino W. Ubiquitination and degradation of CFTR by the E3 ubiquitin ligase MARCH2 through its association with adaptor proteins CAL and STX6. PLoS One 2013;8:e68001.

[41] Xia D, Qu L, Li G, Hongdu B, Xu C, et al. MARCH2 regulates autophagy by promoting CFTR ubiquitination and degradation and PIK3CA-AKT-MTOR signaling. Autophagy 2016;12:1614-30.

[42] Nakamura N, Fukuda H, Kato A, Hirose S. MARCH-II is a syntaxin-6binding protein involved in endosomal trafficking. Mol Biol Cell 2005;16:1696-710.

[43] Cao Z, Huett A, Kuballa P, Giallourakis C, Xavier RJ. DLG1 is an anchor for the E3 ligase MARCH2 at sites of cell-cell contact. Cell Signal 2008;20:73-82.

[44] Fukuda H, Nakamura N, Hirose S. MARCH-III is a novel component of endosomes with properties similar to those of MARCH-II. J Biochem 2006;139:137-45.

[45] Fatehchand K, Ren L, Elavazhagan S, Fang H, Mo X, et al. Toll-like receptor 4 ligands down-regulate fcgamma receptor IIb (FcgammaRIIb) via MARCH3 protein-mediated ubiquitination. J Biol Chem 2016;291:3895-904.

[46] Leclair HM, Andre-Gregoire G, Treps L, Azzi S, Bidere N, Gavard J. The E3 ubiquitin ligase MARCH3 controls the endothelial barrier. FEBS Lett 2016;590:3660-8.

[47] Morokuma Y, Nakamura N, Kato A, Notoya M, Yamamoto Y, et al. MARCH-XI, a novel transmembrane ubiquitin ligase implicated in ubiquitin-dependent protein sorting in developing spermatids. J Biol Chem 2007;282:24806-15.

[48] Bartee E, McCormack A, Fruh K. Quantitative membrane proteomics reveals new cellular targets of viral immune modulators. PLoS Pathog 2006;2:e107.

[49] Nice TJ, Deng W, Coscoy L, Raulet DH. Stress-regulated targeting of the NKG2D ligand Mult1 by a membrane-associated RING-CH family E3 ligase. J Immunol 2010;185:5369-76.

[50] Gu H, Li Q, Huang S, Lu W, Cheng F, Gao P, et al. Mitochondrial E3 ligase March5 maintains stemness of mouse ES cells via suppression of ERK signalling. Nat Commun 2015;6:7112.

[51] Yonashiro R, Ishido S, Kyo S, Fukuda T, Goto E, et al. A novel mitochondrial ubiquitin ligase plays a critical role in mitochondrial dynamics. EMBO J 2006;25:3618-26.

[52] Nakamura N, Kimura Y, Tokuda M, Honda S, Hirose S. MARCH-V is a novel mitofusin 2- and Drp1-binding protein able to change mitochondrial morphology. EMBO Rep 2006;7:1019-22.

[53] Park YY, Lee S, Karbowski M, Neutzner A, Youle RJ, Cho H. Loss of MARCH5 mitochondrial E3 ubiquitin ligase induces cellular senescence through dynamin-related protein 1 and mitofusin 1. J Cell Sci 2010;123:619-26.

[54] Yonashiro R, Sugiura A, Miyachi M, Fukuda T, Matsushita N, et al Mitochondrial ubiquitin ligase MITOL ubiquitinates mutant SOD1 and attenuates mutant SOD1-induced reactive oxygen species generation. Mol Biol Cell 2009;20:4524-30.

[55] Sugiura A, Yonashiro R, Fukuda T, Matsushita N, Nagashima S, et al. A mitochondrial ubiquitin ligase MITOL controls cell toxicity of polyglutamineexpanded protein. Mitochondrion 2011;11:139-46.

[56] Yonashiro R, Kimijima Y, Shimura T, Kawaguchi K, Fukuda T, et al Mitochondrial ubiquitin ligase MITOL blocks S-nitrosylated MAP1B-light chain 1-mediated mitochondrial dysfunction and neuronal cell death. Proc Natl Acad Sci USA 2012;109:2382-7.

[57] Shi HX, Liu X, Wang Q, Tang PP, Liu XY, et al. Mitochondrial ubiquitin ligase MARCH5 promotes TLR7 signaling by attenuating TANK action. PLoS Pathog 2011;7:e1002057.

[58] Yoo YS, Park YY, Kim JH, Cho H, Kim SH, et al. The mitochondrial ubiquitin ligase MARCH5 resolves MAVS aggregates during antiviral signalling. Nat Commun 2015;6:7910.

[59] Foresti O, Ruggiano A, Hannibal-Bach HK, Ejsing CS, Carvalho P. Sterol homeostasis requires regulated degradation of squalene monooxygenase by the ubiquitin ligase Doa10/Teb4. Elife 2013;2:e00953.

[60] Zavacki AM, Arrojo R, Drigo E, Freitas BC, Chung M, Harney JW, et al. The E3 ubiquitin ligase TEB4 mediates degradation of type 2 iodothyronine deiodinase. Mol Cell Biol 2009;29:5339-47.

[61] Goto E, Ishido S, Sato Y, Ohgimoto S, Ohgimoto K, et al. C-MIR a human E3 ubiquitin ligase, is a functional homolog of herpesvirus proteins MIR1 and MIR2 and has similar activity. J Biol Chem 2003;278:14657-68.

[62] Chen R, Li M, Zhang Y, Zhou Q, Shu HB. The E3 ubiquitin ligase MARCH8 negatively regulates IL-1 beta-induced NF-kappaB activation by targeting the IL1RAP coreceptor for ubiquitination and degradation. Proc Natl Acad Sci USA 2012:109:14128-33.

[63] Toyomoto M, Ishido S, Miyasaka N, Sugimoto H, Kohsaka H. Anti-arthritic effect of E3 ubiquitin ligase c-MIR, expression in the joints. Int Immunol 2011:23:177-83.

[64] Kim MH, Rebbert ML, Ro H, Won M, Dawid IB. Cell adhesion in zebrafish embryos is modulated by march 8. PLoS One 2014;9:e94873.

[65] Tada T, Zhang Y, Koyama T, Tobiume M, Tsunetsugu-Yokota Y, et al. MARCH8 inhibits HIV-1 infection by reducing virion incorporation of envelope glycoproteins. Nat Med 2015;21:1502-7. 
[66] Sun Z, Jha HC, Pei YG, Robertson ES. Major histocompatibility complex class II HLA-DRalpha is downregulated by kaposi's sarcoma-associated herpesvirusencoded lytic transactivator RTA and MARCH8. J Virol 2016;90:8047-58.

[67] Ohmura-Hoshino M, Matsuki Y, Aoki M, Goto E, Mito M, et al. Inhibition of MHC class II expression and immune responses by c-MIR. J Immunol 2006;177:341-54.

[68] Hor S, Ziv T, Admon A, Lehner PJ. Stable isotope labeling by amino acids in cel culture and differential plasma membrane proteome quantitation identify new substrates for the MARCH9 transmembrane E3 ligase. Mol Cell Proteomics 2009;8:1959-71.

[69] Hoer S, Smith L, Lehner PJ. MARCH-IX mediates ubiquitination and downregulation of ICAM-1. FEBS Lett 2007;581:45-51.

[70] Yogo K, Tojima H, Ohno JY, Ogawa T, Nakamura N, et al. Identification of SAMT family proteins as substrates of MARCH11 in mouse spermatids. Histochem Cell Biol 2012;137:53-65.

[71] Ohi MD, Kooi CWV, Rosenberg JA, Ren LP, Hirsch JP, et al. Gould. Structura and functional analysis of essential pre-mRNA splicing factor Prp19p. Mol Cell Biol 2005:25:451-60.

[72] Vander Kooi CW, Ohi MD, Rosenberg JA, Oldham ML, Newcomer ME, et al. The Prp19 U-box crystal structure suggests a common dimeric architecture for a class of oligomeric E3 ubiquitin ligases. Biochemistry 2006;45:121-30.

[73] Sanchez DJ, Coscoy L, Ganem D. Functional organization of MIR2, a novel viral regulator of selective endocytosis. J Biol Chem 2002;277:6124-30.

[74] Bourgeois-Daigneault MC, Thibodeau J. Autoregulation of MARCH1 expression by dimerization and autoubiquitination. J Immunol 2012;188:4959-70.

[75] Bourgeois-Daigneault MC, Thibodeau J. Identification of a novel motif that affects the conformation and activity of the MARCH1 E3 ubiquitin ligase. J Cell Sci 2013;126:989-98.

[76] Shin JS, Ebersold M, Pypaert M, Delamarre L, Hartley A, Mellman I. Surface expression of MHC class II in dendritic cells is controlled by regulated ubiquitination. Nature 2006;444:115-8.

[77] Means RE, Ishido S, Alvarez X, Jung JU. Multiple endocytic trafficking pathways of MHC class I molecules induced by a herpesvirus protein. EMBO J 2002;21:1638-49.

[78] Cresswell P, Bangia N, Dick T, Diedrich G. The nature of the MHC class peptide loading complex. Immunol Rev 1999;172:21-8.

[79] Cosson P, Bonifacino JS. Role of transmembrane domain interactions in the assembly of class II MHC molecules. Science 1992;258:659-62.

[80] King G, Dixon AM. Evidence for role of transmembrane helix-helix interactions in the assembly of the class II major histocompatibility complex. Mol Biosyst 2010;6:1650-61.

[81] Dixon AM, Drake L, Hughes KT, Sargent E, Hunt D, et al. Differential transmembrane domain GXXXG motif pairing impacts major histocompatibility complex (MHC) class II structure. J Biol Chem 2014:289:11695-703.

[82] Drake LA, Drake JR. A triad of molecular regions contribute to the formation of two distinct MHC class II conformers. Mol Immunol 2016;74:59-70.

[83] Kleiger G, Eisenberg D. GXXXG and GXXXA motifs stabilize FAD and NAD(P)binding rossmann folds through $\mathrm{C}($ alpha $)-\mathrm{H}$. . O hydrogen bonds and van der waals interactions. J Mol Biol 2002;323:69-76.
[84] Sato T, Kienlen-Campard P, Ahmed M, Liu W, Li H, et al. Inhibitors of amyloid toxicity based on beta-sheet packing of Abeta 40 and Abeta42. Biochemistry 2006;45:5503-16.

[85] Decock M, Stanga S, Octave JN, Dewachter I, Smith SO, et al. Glycines from the APP GXXXG/GXXXA transmembrane motifs promote formation of pathogenic abeta oligomers in cells. Front Aging Neurosci 2016;8:107.

[86] Russ WP, Engelman DM. The GxxxG motif: a framework for transmembrane helix-helix association. J Mol Biol 2000;296:911-9.

[87] Senes A, Gerstein M, Engelman DM. Statistical analysis of amino acid patterns in transmembrane helices: the GxxxG motif occurs frequently and in association with beta-branched residues at neighboring positions. J Mol Biol 2000;296:921-36.

[88] Teese MG, Langosch D. Role of GxxxG motifs in transmembrane domain interactions. Biochemistry 2015;54:5125-35.

[89] Cymer F, Veerappan A, Schneider D. Transmembrane helix-helix interactions are modulated by the sequence context and by lipid bilayer properties. Biochim Biophys Acta 2012;1818:963-73.

[90] Doura AK, Kobus FJ, Dubrovsky L, Hibbard E, Fleming KG. Sequence context modulates the stability of a GxxxG-mediated transmembrane helix-helix dimer. J Mol Biol 2004;341:991-8.

[91] Melnyk RA, Kim S, Curran AR, Engelman DM, Bowie JU, Deber CM. The affinity of GXXXG motifs in transmembrane helix-helix interactions is modulated by long-range communication. J Biol Chem 2004;279:16591-7.

[92] Johnson RM, Rath A, Deber CM. The position of the gly-xxx-gly motif in transmembrane segments modulates dimer affinity. Biochem Cell Biol 2006;84:1006-12.

[93] Leung JH, Schurig-Briccio LA, Yamaguchi M, Moeller A, Speir JA, et al. Structural biology. Division of labor in transhydrogenase by alternating proton translocation and hydride transfer. Science 2015;347:178-81.

[94] Lee C, Yashiro S, Dotson DL, Uzdavinys P, Iwata S, et al. Crystal structure of the sodium-proton antiporter NhaA dimer and new mechanistic insights. J Gen Physiol 2014:144:529-44.

[95] Morth JP, Pedersen BP, Toustrup-Jensen MS, Sorensen TL, Petersen J, et al. Crystal structure of the sodium-potassium pump. Nature 2007;450:1043-9.

[96] Monne M, Nilsson I, Elofsson A, von Heijne G. Turns in transmembrane helices: determination of the minimal length of a helical hairpin and derivation of a fine-grained turn propensity scale. J Mol Biol 1999;293:80714.

[97] Kim SH, Park YY, Yoo YS, Cho H. Self-clearance mechanism of mitochondrial E3 ligase MARCH5 contributes to mitochondria quality control. FEBS J 2016;283:294-304.

[98] Krogh A, Larsson B, von Heijne G, Sonnhammer EL. Predicting transmembrane protein topology with a hidden markov model: application to complete genomes. J Mol Biol 2001;305:567-80.

[99] Kumar S, Stecher G, Tamura K. MEGA7: molecular evolutionary genetics analysis version 7.0 for bigger datasets. Mol Biol Evol 2016;33:1870-4.

[100] Sievers F, Wilm A, Dineen D, Gibson TJ, Karplus K, et al. Fast, scalable generation of high-quality protein multiple sequence alignments using clustal omega. Mol Syst Biol 2011;7:539.

[101] Waterhouse JB, Procter DM, Martin M. Jalview version 2-a multiple sequence alignment editor and analysis workbench. Bioinformatics 2009;25:1189-91. 